\title{
EGFR mutation positive non-small cell lung cancer: can we identify predictors of benefit from immune checkpoint inhibitors
}

\author{
Ying Wang, Peter Ellis \\ Department of Oncology, McMaster University, Hamilton, ON, Canada \\ Correspondence to: Peter Ellis, MBBS, MMed, PhD. Juravinski Cancer Centre, 699 Concession Street, Hamilton, Ontario L8V 5C2, Canada. \\ Email: ellisp@hhsc.ca. \\ Provenance: This is a Guest Editorial commissioned by Section Editor Jianrong Zhang, M.P.H. candidate (George Warren Brown School of Social \\ Work, Washington University in St. Louis, St. Louis, USA. Academic relationship to: Department of Thoracic Surgery \& Oncology, First Affiliated \\ Hospital of Guangzhou Medical University, Guangzhou Institute of Respiratory Disease, Guangzhou, China). \\ Comment on: Haratani K, Hayashi H, Tanaka T, et al. Tumor Immune Microenvironment and Nivolumab Efficacy in EGFR Mutation-Positive Non- \\ Small Cell Lung Cancer Based on T790M Status after Disease Progression During EGFR-TKI Treatment. Ann Oncol 2017;28:1532-9.
}

Submitted Jul 18, 2017. Accepted for publication Jul 31, 2017.

doi: $10.21037 / \mathrm{atm} .2017 .08 .14$

View this article at: http://dx.doi.org/10.21037/atm.2017.08.14

Monoclonal antibodies directed against the programmed death-1 (PD-1) receptor have demonstrated consistent survival improvement in comparison to second-line chemotherapy with docetaxel, in patients with advanced non-small cell lung cancer (NSCLC), across multiple histologic subtypes. However, this benefit is less clear in some subgroups of patients, including never smokers and patients with tumors harboring activating driver mutations, such as mutations of the epidermal growth factor receptor $(E G F R)$ gene. Sub-group analyses of several phase III clinical trials comparing the immuno-oncology (IO) agents, nivolumab, pembrolizumab, or atezolizumab, with docetaxel, have failed to demonstrate superior efficacy of IO in patients with EGFR mutated tumors when compared to standard of care chemotherapy (1-3).

However, EGFR mutations are heterogeneous in nature. In treatment naive patients, differences in the type of EGFR mutation are known to impact on the efficacy of therapy (4). Heterogeneity also exists in the mechanisms by which tumors develop resistance to EGFR directed therapy (5). More than $50 \%$ of patients demonstrating resistance to first-line EGFR tyrosine kinase inhibitors (TKIs) develop T790M secondary mutations (6). Multiple mechanisms of resistance occur in the remaining patients including overexpression of the mesenchymal to epithelial transition (MET) and insulin-like growth factor receptor $(I G F-R)$ genes and even transformation to small cell lung cancer in some patients. It is unclear how these resistance mechanisms may impact on subsequent response to IO therapy in this group of patients. It is in this context that Haratani and colleagues report on biomarker discovery research to identify predictive biomarkers for IO therapy in patients with EGFR mutated NSCLC (7).

The current study, reported by Haratani and colleagues, explored the impact of T790M mutation status on overall response rate (ORR) and progression free survival (PFS) from nivolumab, to identify additional potential predictive biomarkers for response to IO, in patients with EGFR mutated NSCLC (7). They report on a cohort of 25 NSCLC patients with activating mutations of the EGFR gene, previously treated with an EGFR TKI, receiving therapy with nivolumab, where T790M mutation status was known. Tissue from formalin fixed paraffin embedded (FFPE) blocks obtained following the development of EGFR TKI resistance was analyzed where available. This included assessment of immunohistochemistry (IHC) staining for PD-L1 expression, tumor-infiltrating lymphocyte (TIL) density, CD8, CD4, and FOXP3 expression, as well as whole exome sequencing (WES) in extracted DNA samples, to quantify the burden of nonsynonymous tumor mutations. The analyses of these data looked at the predictive value of these putative biomarkers on ORR and PFS, according to T790M tumor mutation status. Lastly, the authors mention IHC analysis on a second cohort of 60 patients with EGFR mutation positive NSCLC, who did not receive therapy with nivolumab 
(cohort B).

It is important to realize that this is a very small sample of patients and statistical comparisons between groups is not particularly informative, because they are greatly underpowered. Among the 25 patients studied, eight had tumors positive for T790M mutation, which is somewhat lower than expected. The ORR to nivolumab in the entire cohort of 25 patients was $20 \%$. The ORR was higher among patients who were T790M negative (24\% in T790M negative patients, $13 \%$ in EGFR T790M positive patients), although this difference was not statistically different $(\mathrm{P}=1.000)$. Similarly, the disease control rate (DCR) was higher in patients with T790M negative tumors than T790M positive tumors (DCR, 47\% vs. $13 \%$ respectively, $\mathrm{P}=0.182$ ). The median PFS was 1.4 months (mos) for the entire cohort, and there was little difference between the median PFS of patients with T790M negative tumors (2.1 mos) and T790M positive tumors (1.3 mos, $\mathrm{P}=0.099)$. Interestingly, the hazard ratio (HR) for PFS was 0.48 [95\% confidence interval (CI), 0.20-1.24] raising the potential for longer PFS among patients with T790M mutation negative tumors. Median overall survival (OS) had not been reached in the study with a median follow-up of 7.3 mos. Although it favored patients with T790M negative tumors (HR, 0.38; 95\% CI, 0.09-1.65), this difference was not significant.

Information was missing for many patients in the biomarker discovery analysis. PD-L1 status was known in only 15 of 25 patients and only 14 of 25 patients had evaluable TIL density scores. Only 9 of 25 patients had material available for WES. There appears to be an association between the likelihood of response to therapy and tumor PD-L1 expression. ORR increases as PDL1 expressions increase from $\geq 1 \%$, to $\geq 10 \%$, to $\geq 50 \%$, corresponding to ORR of $38 \%(n=8), 75 \%(n=4)$, and $100 \%$ $(n=2)$ respectively. PFS also favored patients with tumors showing any PD-L1 positivity $\geq 1 \%$ (2.1 vs. 1.3 mos; HR, $0.37)$. In cohort $B(n=60)$, the proportion of patients whose tumors demonstrated any PD-L1 positivity was similar between T790M mutation negative and positive tumors. However, there appears to be an association between higher levels of PD-L1 expression and T790M mutation negative tumors. A higher proportion of tumors demonstrated staining for PD-L1 $\geq 10 \%$ and $\geq 50 \%$, in T790M negative than T790M positive patients.

Biomarker analysis of TIL density was also explored. Of the three immunotypes assessed, only the CD8 positive TIL counts were higher in nivolumab responders when compared to non-responders $(\mathrm{P}=0.024)$, whereas CD4 positive and FOXP3 positive TIL densities were similar in both groups. PFS assessments based on differences in TIL densities did not yield any statistically significant results in this study. Among the nine patients assessed with WES, the three patients with response to IO demonstrated a significantly higher non-synonymous mutation rate compared to the six who did not respond $(\mathrm{P}=0.038)$. This did not differ by T790M status $(\mathrm{P}=0.710)$.

The findings by Haratani and colleagues shed light on the efficacy of IO agents in NSCLC patients with EGFR mutations. Data from randomized trials comparing nivolumab, pembrolizumab, or atezolizumab with docetaxel have failed to show a survival improvement in patients with an EGFR mutation receiving the $\mathrm{IO}$ agent $(8,9)$. However, these findings are not entirely consistent across studies. The ORR and PFS seen in this trial with nivolumab are comparable to those observed with IOs in randomized trials of NSCLC patients in the second line setting (1-3). Interestingly in this study, both ORR and DCR is numerically higher in the T790M negative population, than the T790M positive population. Therefore, the mechanism of secondary resistance to EGFR directed therapy may provide some insight into the apparent lower efficacy of IO agents in NSCLC patients harboring EGFR mutations. OS data in this trial was immature. However, the data suggest that survival in T790M mutation negative patients treated with nivolumab may be greater than T790M mutation positive patients. Variability in the rate of T790M mutations in the second-line randomized trials of IO, might be implicated in the difference in efficacy of IO agents in EGFR mutated NSCLC. This hypothesis requires further exploration in prospective studies where T790M mutation status is known.

The data on PFS is more difficult to interpret. The observed PFS in the data from Haratani et al. (7) is reflective of published data from randomized trials (1-3). The majority of the patients progressed early, with a median PFS of 1.5 mos, independent of T790M status. However, actuarial analysis of PFS suggested longer PFS among patients with T790M mutation negative tumors (HR, 0.48; 95\% CI, 0.20-1.21). It may be more informative in future research to look at PFS at 6 or 12 mos to identify the EGFR mutation positive patients who respond to IO, and determine factors associated with durable response to therapy.

There is some preclinical work to support the findings from Haratani and colleagues. Mullerian and cell-line studies suggest increases in PD-L1 staining correspond 
to increased response to IO in EGFR mutation positive patients $(10,11)$. There was a trend towards higher ORR with increased PD-L1 expression in this study, although the small sample size of the study limits the power to confidently demonstrate this. The expected distribution of PD-L1 expression in unselected NSCLC patients is around $50-60 \%$ PD-L1 positive, and 30\% of all NSCLC are highly positive, as defined by PD-L1 staining of $\geq 50 \%$ of tumor cells. In the current study by Haratani et al. (7), we see a slightly lower rate of any PD-L1 expression $\geq 1 \%$ among patients with $E G F R$ mutations, with much fewer patients than expected, demonstrating high PD-L1 expression $\geq 50 \%$ (2/9 in the T790M negative group, and $0 / 6$ in the T790M positive group). Since higher PD-L1 staining tends to correlate with better response to IO, this may provide a glimpse into the mechanism behind lower response to IO in this population of EGFR mutated NSCLC.

Data on non-synonymous mutation burden (NMB) in this study is very limited but is consistent with other data in NSCLC. Earlier studies suggested that the NMB is associated with improved objective response rates, and durable clinical benefit, thus this study provides support to existing knowledge in the area (12). In general, NSCLC has on average a NMB of 6.86 per megabase $(\mathrm{Mb})$, or $200 \mathrm{NMBs}$ per tumor sample with a wide range of 11 to $1,192 \mathrm{NMB}$ per sample $(12,13)$. The samples in the current study had both a much lower NMB/ sample, and $\mathrm{NMB} / \mathrm{Mb}$ than data from unselected populations of NSCLC patients, likely as a reflection of the different molecular profiles between EGFR mutation positive tumors $v s$. smoking associated lung cancers.

The current study suggests that EGFR mutated NSCLC tumors have lower levels of PD-L1 expression and NMB. Since both tumor mutational burden and higher PD-L1 levels have potential predictive value for response to IO, this study may highlight a key reason underlying the poorer efficacy to IO observed among patients with EGFR mutated, compared to non-EGFR mutated NSCLC in previous phase III trials. In the second-line setting, some inconsistency has been observed between studies in the association of PD-L1 expression and improved OS from IO compared with docetaxel $(1,2)$. Therefore, PD-L1 expression should generally not be used to select patients for IO in the secondline setting. However, given the findings by Haratani and colleagues, PD-L1 expression may prove useful in selecting patients with EGFR mutated NSCLC for IO vs. secondline chemotherapy, although this requires validation in prospective trials in these populations.

The observations on TILs in this study is thought provoking, but requires additional research. TILs may be a prognostic factor in multiple cancers independent of treatment, and is one of multiple biomarkers being actively assessed in conjunction with PD-L1 expression. Thus, the significance of higher CD8+ve TIL count in nivolumab responders than non-responders may guide further research for predictive biomarker testing. In addition to TIL density, including other potentially predictive biomarkers such as T-cell receptor clonality, or neoantigen burden, may also yield interesting hypotheses for further research in an EGFR mutation positive population (14).

Overall, this study highlights several interesting hypotheses involving EGFR mutation positive NSCLC patients and IO which are in need of further exploration. The lack of survival benefit from IO therapy compared with docetaxel, among patients with EGFR mutated NSCLC, has led some to suggest that these patients may not benefit from IO therapy. We would not agree with this view, but think that factors such as the presence or absence of T790M mutation, PD-L1 expression, and tumor mutation burden may all help to refine the selection of EGFR mutation positive NSCLC patients, for therapy with IO agents. Although we cannot use the information presented in this study to direct therapy for these patients, it does provide promising avenues of research for prospective trials that may help shed light on the area. The multiple emerging concepts in lung cancer diagnostics such as TIL density, tumor mutation burden assessment, and PD-L1 testing in this unique population are all areas requiring further clinical validation.

The combination of numerically higher ORR, and PFS as well as supporting exploratory biomarker assessment helps one hypothesize that EGFR TKI resistance in the absence of a T790M mutation may be due to a higher burden of molecular abnormalities, and these patients may potentially benefit more from IO therapy than patients with a T790M mutation. Thus, trials assessing the outcomes of responses to IO in patients with EGFR activating mutations, especially in those who do not harbor further targetable resistant mutations such as in the T790M, MET, and HER2 populations, should receive the highest priority.

\section{Acknowledgements}

None.

\section{Footnote}

Conflicts of Interest: Dr. P Ellis has received honoraria for 
speaking from Boehringer-Ingelheim, Pfizer and Novartis. The other author has no conflicts of interest to declare.

\section{References}

1. Borghaei H, Paz-Ares L, Horn L, et al. Nivolumab versus Docetaxel in Advanced Nonsquamous Non-Small-Cell Lung Cancer. N Engl J Med 2015;373:1627-39.

2. Rittmeyer A, Barlesi F, Waterkamp D, et al. Atezolizumab versus docetaxel in patients with previously treated non-small-cell lung cancer (OAK): a phase 3, openlabel, multicentre randomised controlled trial. Lancet 2017;389:255-65.

3. Herbst RS, Baas P, Kim DW, et al. Pembrolizumab versus docetaxel for previously treated, PD-L1-positive, advanced non-small-cell lung cancer (KEYNOTE-010): a randomised controlled trial. Lancet 2016;387:1540-50.

4. Yang JC, Wu YL, Schuler M, et al. Afatinib versus cisplatin-based chemotherapy for EGFR mutation-positive lung adenocarcinoma (LUX-Lung 3 and LUX-Lung 6): analysis of overall survival data from two randomised, phase 3 trials. Lancet Oncol 2015;16:141-51.

5. Oxnard GR, Arcila ME, Sima CS, et al. Acquired resistance to EGFR tyrosine kinase inhibitors in EGFRmutant lung cancer: distinct natural history of patients with tumors harboring the T790M mutation. Clin Cancer Res 2011;17:1616-22.

6. Kobayashi S, Boggon TJ, Dayaram T, et al. EGFR mutation and resistance of non-small-cell lung cancer to gefitinib. N Engl J Med 2005;352:786-92.

Cite this article as: Wang Y, Ellis P. EGFR mutation positive non-small cell lung cancer: can we identify predictors of benefit from immune checkpoint inhibitors. Ann Transl Med 2017;5(21):424. doi: 10.21037/atm.2017.08.14
7. Haratani K, Hayashi H, Tanaka T, et al. Tumor Immune Microenvironment and Nivolumab Efficacy in EGFR Mutation-Positive Non-Small Cell Lung Cancer Based on T790M Status after Disease Progression During EGFRTKI Treatment. Ann Oncol 2017;28:1532-9.

8. Lee CK, Man J, Lord S, et al. Checkpoint Inhibitors in Metastatic EGFR-Mutated Non-Small Cell Lung Cancer-A Meta-Analysis. J Thorac Oncol 2017;12:403-7.

9. Ellis PM, Vella ET, Ung YC. Immune Checkpoint Inhibitors for Patients With Advanced Non-Small-Cell Lung Cancer: A Systematic Review. Clin Lung Cancer 2017;18:444-59.e1.

10. Akbay EA, Koyama S, Carretero J, et al. Activation of the PD-1 pathway contributes to immune escape in EGFRdriven lung tumors. Cancer Discov 2013;3:1355-63.

11. Azuma K, Ota K, Kawahara A, et al. Association of PDL1 overexpression with activating EGFR mutations in surgically resected nonsmall-cell lung cancer. Ann Oncol 2014;25:1935-40.

12. Rizvi NA, Hellmann MD, Snyder A, et al. Cancer immunology. Mutational landscape determines sensitivity to PD-1 blockade in non-small cell lung cancer. Science 2015;348:124-8.

13. Cancer Genome Atlas Research Network. Comprehensive molecular profiling of lung adenocarcinoma. Nature 2014;511:543-50.

14. Gibney GT, Weiner LM, Atkins MB. Predictive biomarkers for checkpoint inhibitor-based immunotherapy. Lancet Oncol 2016;17:e542-51. 